\title{
A family of models to study the growth of Haloferax mediterranei in different conditions
}

\author{
Y. Villacampa ${ }^{1}$, F. García-Alonso ${ }^{1}$, J. A. Reyes ${ }^{1}$, \\ R. Martínez-Espinosa ${ }^{2} \&$ M. J. Bonete ${ }^{2}$ \\ ${ }^{I}$ Departamento de Matemática Aplicada, Universidad de Alicante, Spain \\ ${ }^{2}$ Departamento de Agroquímica y Bioquímica, Universidad de Alicante, \\ Spain
}

\begin{abstract}
Haloferax mediterranei is a denitrifying halophilic archaeon able to grow with nitrite as the sole nitrogen source for growth in an assimilatory process under aerobic conditions. This haloarchaeon can also reduce nitrite in a respiratory process, where nitrite is the electron acceptor when oxygen conditions are limited. Due to this capability, Haloferax mediterranei could be applied in salted water bioremediation processes with the purpose of repairing the damage caused by the excessive use of fertilizers in agricultural activities.

In this paper a family of different mathematical models has been generated to allow the study and the prediction of $H f x$. mediterranei growth in high salt media with different nitrite concentrations. The relation between the growth and some variables are studied, for example nitrite concentration $(\mathrm{N})$, oxygen concentration $(\mathrm{O})$ and the time of growth $(\mathrm{NH})$. This approach will allow us to analyse future $H f x$. mediterranei uses as agent for bioremediation processes. Keywords: denitrification, Haloferax, bioremediation, modelling, stability.
\end{abstract}

\section{Introduction}

Denitrification is the reduction of nitrate or nitrite to gaseous nitrogen oxides. This pathway occurs mainly in bacteria and is used by most denitrifiers to support respiratory growth under anaerobic conditions [17]. Denitrification produces nitrogen loss in agricultural soils, and emitted $\mathrm{N}_{2} \mathrm{O}$ destroys the ozone layer and contributes to global warming. Deterioration of quality of inland and coastal waters is a serious environmental problem. Of particular concern is the 
wastewater containing organic nitrogen [6]. Nitrogen compounds can be removed from wastewater by a variety of physicochemical and biological processed. However, biological nitrogen removal is more effective and relatively inexpensive, by this reason it has been widely adopted in favour of the physicochemical processes [1]. In this way, denitrification could fill an important function in waste treatment by removing excess nitrogen in local environments and by anaerobically degrading organic pollutants. Nevertheless, high salt concentrations exert adverse effects on the metabolic pathway mentioned above [5]. By this reason, extreme microorganisms (mainly halophiles) have focused the scientific attention in the last few years.

Extremely halophilic archaea (haloarchaea) generally grow heterotrophically under aerobic conditions in hypersaline environments, although they possess facultative anaerobic capabilities [4]. It has been demonstrated that $H f x$. mediterranei grows under anaerobic conditions using nitrate as terminal electron acceptor [8], and we observed the induction of respiratory nitrate reductase (Nar) activity in these cultures. These results agree with other studies in which nitratereducing and denitrifying activities are induced under oxygen-limiting conditions only in the presence of nitrate [17]. Recently, it has been shown that $H f x$. mediterranei also grows in the presence of nitrite concentrations as high as 40 $\mathrm{mM}$, although the growth is slightly slow if it is compared with the growth rate observed in rich culture media or minimal culture media under aerobic conditions [9]. Nitrite is present as a natural component of the nitrogen cycle in freshwater ecosystems, however, its concentrations are increasing in freshwater environments as a consequence of several anthropogenic sources such as effluents from industries producing metals, dyes and celluloids, urban sewage effluents and aquaculture [2]. This nitrogen source (nitrite) is very toxic to aquatic animals, microorganisms, even to humans, because nitrite is able to oxidise the iron of the hemoglobin molecule to methemoglobin. The last molecule is not able to transport oxygen causing anoxia and death [2].

$H f x$. mediterranei could be applied in salted water bioremediation processes with the purpose to repair the damage caused by the excessive use of fertilizers in agricultural activities. This application could be beneficial in regions such as Comunidad Valencia or Murcia (Spain), where the water tables contain high nitrate and nitrite concentrations due to fertilization practices [7]. In this work, using the experimental data, it has been carried out several mathematical studies to predict the $H f x$. mediterranei growth in high salt media with different nitrite concentrations. It has been used methodologies to generate families of mathematical models, selecting those suitable models to explain the Haloferax mediterranei behaviour under certain conditions.

\section{Methodology}

\subsection{Physiological experiments}

Hfx. mediterranei (ATCC 33500T) was grown in a minimal culture medium $(\mathrm{pH} 7.3)$ with different nitrite concentrations $\left(5-45 \mathrm{mM} \mathrm{NO}_{2}^{-}\right)$as sole nitrogen source as described before [9]. The oxygen concentration $\left[\mathrm{O}_{2}\right]$ was $100 \%$ when 
the medium was inoculated with $5 \mathrm{ml}$ of a seed culture grown with $20 \mathrm{mM}$ nitrite as nitrogen source. $H f x$. mediterranei was grown at $37{ }^{\circ} \mathrm{C}$ in a $2 \mathrm{~L}$ flask in a Biostat B fermentor (B. Braun Biotech International). The $\left[\mathrm{O}_{2}\right]$ was estimated with a calibrated $\mathrm{pO}_{2}$ electrode (Metller) during $H f x$. mediterranei growth. The growth was monitored by measuring the optical density (OD) at $600 \mathrm{~nm}$ using a Biofotometer (Eppendorf). Nitrite was quantified using the diazo coupling method [10]. $2 \mathrm{ml}$ aliquots of the cultures were taken during the growth period in order to determine all cited parameters.

\subsection{Mathematical studies}

There are several methodologies to mathematical formulation of the relations obtained from experimental data. The methodologies implemented in software such as SPLUS and SPSS share the common characteristic of determining simple and multiple linear relations in a similar manner [11,12]. It is also possible to obtain nonlinear relations so that for each execution, a type of equation is proposed, with coefficients calculated by applying the least squares method. To the automatic search among different models, we must cite $[3,15]$, which present the Modelhss methodology, with which families of nonlinear models can be obtained in a formal language generated from different orders of vocabularies. The equations obtained in Modelhss are linear combinations of functions defined on the basis of vocabularies, and their statistical treatment is reduced to the multiple linear cases, as their parameters are linear. The methodology developed in [13] generates families of mathematical models with nonlinear parameters, and includes the study of linear models, based on the experimental data of the intervening variables.

\section{Results and discussion}

Previous physiological experimental analysis have revealed that $H f x$. mediterranei could be an excellent model to establish new salted water bioremediation processes [9], because this haloarchaeon is able to reduce high nitrate and nitrite concentrations in presence of high salt concentrations. This ability is quite interesting from a biotechnological point of view because most of the denitrifying microorganisms are unable to develop these reactions under salted conditions [5]. We have analysed different mathematical models to predict the $H f x$. mediterranei growth in cultures with high nitrite concentration, using methodologies studied in [11-16]. The followed mathematical expression has been found as the better model to predict Hfx. mediterranei growth under the cited conditions in methodology.

It is proposed as a better model

$$
C=-1.714 e^{0.023 N}+0.003 O x+4.259, \quad R^{2}=0.9
$$

So it can write, 


$$
N=\frac{1}{0.023} \ln \left(\frac{1}{1.714}(-C+0.003 O x+4.259)\right)
$$

It has been studied the stability of the models against perturbations in experimental data, applying the methodology implemented in $[14,16]$. It can be observed that for perturbations of $10 \%$ and $20 \%$ in the experimental data, the model is stable (Figure 1). In this figure is observed the variation that it is produced in the model when it has been carried out perturbations of $10 \%$ and $20 \%$ in experimental data.

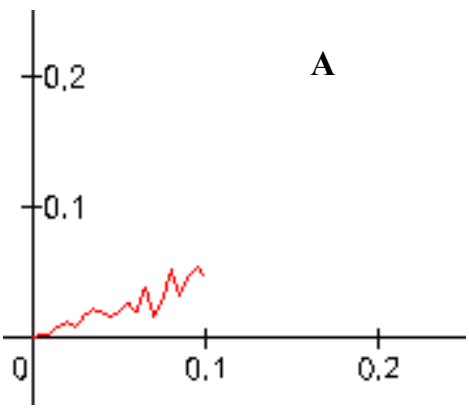

Perturbations in experimental data

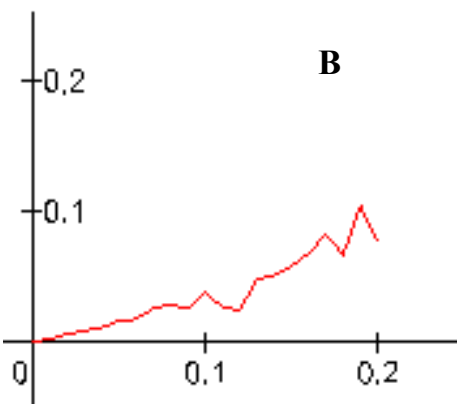

Perturbations in experimental data

Figure 1: Stability of the models. A) the perturbation of experimental data was $10 \%$ and in B) the perturbation of experimental data was $20 \%$.

Using the model and Eq. (2) it has been carried out an estimate of the nitrite present into the culture media during the growth at different times. These results can be observed in the Figure 2.

It can be deduced that the behaviour inferred for the growth of $H f x$. mediterranei in $45 \mathrm{mM}$ nitrite is similar to the behaviour experimentally observed and described previously for $40 \mathrm{mM}$ nitrite (Figure 3) [9].

The results obtained in (Figure 3) show that $H f x$. mediterranei grows in the presence of nitrite concentrations as high as $40 \mathrm{mM}$. As can be seen in the OD curve of the figure 3 , two different metabolic processes can be distinguish; i) the growth is especially slow during the first 10 days, a period of time in which the $\left[\mathrm{O}_{2}\right]$ is higher than $2 \%$. This phase of growth correspond to the aerobic nitrite reduction pathway where nitrite is used as nitrogen source for growth. When the oxygen is completely consumed, the rate of growth increased substantially, indicating that nitrite present in the culture media could act as nitrogen source and as the terminal electron acceptor under anoxic conditions. The nitrite present in the culture media was removed during the growth of $H f x$. mediterranei, in fact, at the final of the stationary phase of growth $\left(\mathrm{OD}_{600}=2.2\right)$, only $20 \%$ of the nitrite present in the culture was not eliminated. As it has been described before, these metabolic pathways are usually inhibited under salted conditions. So it is possible to think that this haloarchaeon could be applied in water bioremediation 
Hfx. mediterranei growth in $45 \mathrm{mM}$ nitrite

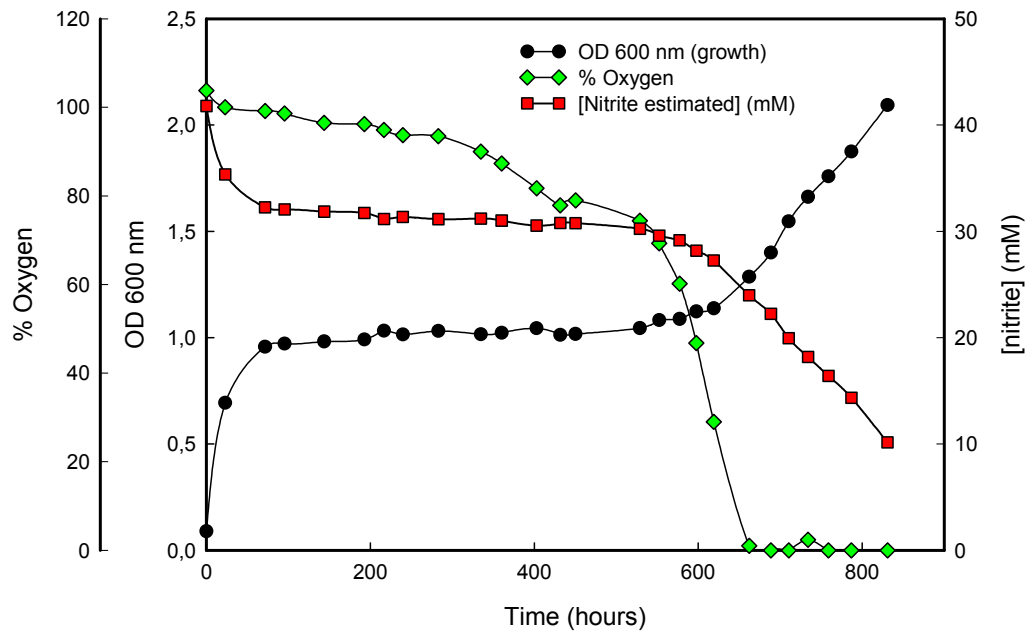

Figure 2: The prediction of $H f x$. mediterranei growth in a minimal culture medium with nitrite as the sole nitrogen source. OD at $600 \mathrm{~nm}$; nitrite concentration in the culture medium; percentage of $\mathrm{O}_{2}$.

Hfx. mediterranei growth in $40 \mathrm{mM}$ nitrite

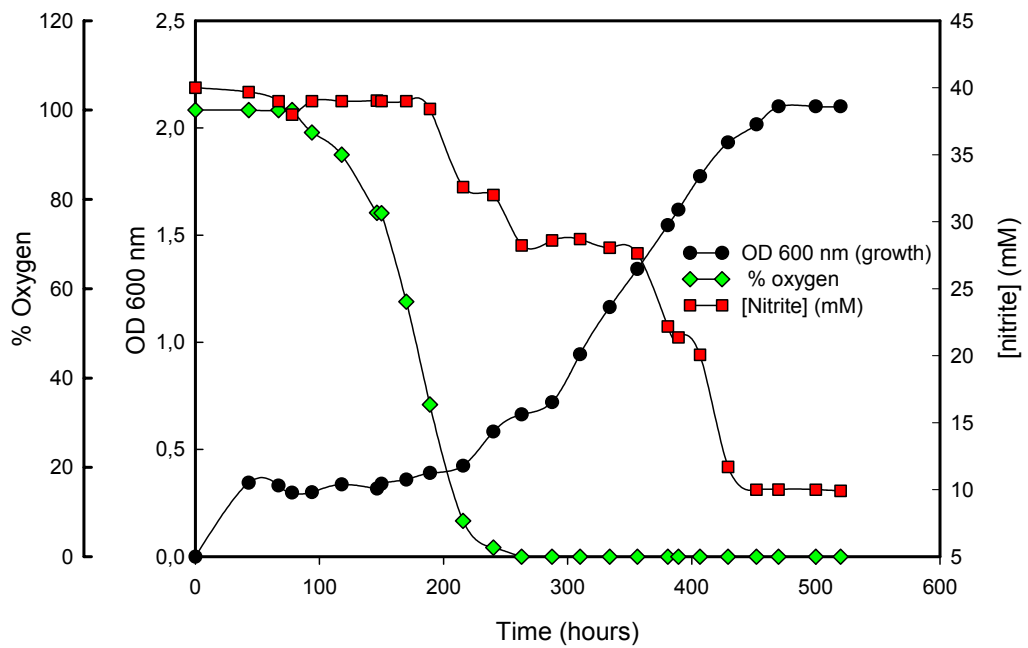

Figure 3: Experimental $H f x$. mediterranei growth in a minimal culture medium with nitrite as the sole nitrogen source. OD at $600 \mathrm{~nm}$; nitrite concentration in the culture medium; percentage of $\mathrm{O}_{2}$. 
processes with the purpose to repair the damage caused by the excessive use of fertilizers in agricultural activities. Besides, it has been demonstrated that $H f x$. mediterranei growth can be predicted with mathematical approaches, which make easier the applications of this haloarchaeon in salted water treatments.

\section{Conclusions}

With the mathematical models obtained it has been able to compare and predict the experimental physiological results previously described from $H f x$. mediterranei growth taken in account different parameters such as nitrogen source or oxygen concentration. The models obtained allow us to analyse and validate the experimental results carried out previously [9].

Our aim is to obtain new sets of experimental data under similar and different conditions to those consider in this article with the objective to generate new mathematical model. With this kind of studies, we contribute to the modern biocatalysts knowledge which is achieving new advances in environmental and healthy fields (using enzymatic or whole cells bioremediation).

\section{Acknowledgements}

This work was supported in part by funds from AE/07/091 (MJB) and AE/07/074 (YVE) from Generalitat Valenciana.

The authors thank Dr. F. Verdu and Dr Y. Villacampa for providing the mathematical models $[13,15]$ and the stability of the models $[14,16]$.

\section{References}

[1] Ahn, YH. Sustainable nitrogen elimination biotechnologies: a review. Process Biochemistry, 41, 1709-1721, (2006).

[2] Alonso, A. and Camargo J.A. Toxicity of nitrite to three species of freshwater invertebrates. Environmental Toxicology, 90-94 (2006)

[3] Cortés, M. ; Villacampa, Y.; Mateu, \& Usó,. J.L. 'A new methodology for modelling highly structured systems' Environmental Modelling Software 15, pp 461-470, (2000).

[4] DasSarma, S., and P. Arora. Halophiles, p. 458-466. In Encyclopedia of life sciences, vol. 8. Nature Publishing Group, London, United Kingdom, (2002).

[5] Kargi, F. and Uygur, A. Biological treatment of saline wastewater in a rotting biodisc contactor by using halophilic organisms. Bioprocess Engineering, 17, 81-85 (1997)

[6] Khin, T. and Annachhatre, A.P. Novel microbial nitrogen removal processes. Biotechnology advances, 22, 519-532 (2004)

[7] Legaz, F. \& Primo-Millo, E. Influencia de la fertilización nitrogenada en la contaminación por nitratos de las aguas subterráneas. Levante Agrícola 318, 4-15, (1992). 
[8] Lledó, B., Martínnez-Espinosa, R.M., Marhuenda-Egea, F.C. and Bonete, M.J. Respiratory nitrate reductase from haloarchaeon Haloferax mediterranei: biochemical and genetic analysis. Biochim. Biophys. Acta 1674, 50-59, (2004).

[9] Martínnez-Espinosa, R.M.; Richardson, D.J.; Butt, J.N. \& Bonete, M.J. Respiratory nitrate and nitrite pathway in the denitrifier haloarchaeon Haloferax mediterranei. Biochemical Society Transactions 34, part 1, 115 117, (2006).

[10] Snell, C.D. \& Snell, C.T. Colorimetric Methods of Analysis, vol. 2, pp. 802-807, Van Nostrand, New York, (1949).

[11] S-Plus 2000. Guide to statistics volume 1,2. Mathsoft, Inc (2000).

[12] Spss. Inc. Software, Chicago, USA (1999).

[13] Verdu, F \& Villacampa, Y. A Computational algorithm for the multiple generation of nonlineal mathematical models and stability study. Advances in Engineering Software. In Press.

[14] Verdu, F \& Villacampa, Y. A computer program for a Monte Carlo analysis of sensitivity in equations of environmental modelling obtained from experimental data. Advances in Engineering Software. Vol. 33, N 6. pp.351-359, (2002).

[15] Villacampa, Y.; Cortés, M.; Vives, F. \& Castro, M.A. 'A new computational algorithm to construct mathematical models.' Ecosystems and sustainable development II Ed. WIT Press (1999).

[16] Villacampa, Y; Verdu, F \& Pérez, A. A Stability theory for model systems. Kybernetes. Vol.36, No .5-10.pp. 1-23, (2007).

[17] Zumft, WG. Cell biology and molecular basis of denitrification. Microbiol Mol Biol Rev. 61(4):533-616, (1997). 